\title{
STEROID-INDUCED PEDIATRIC DIABETES MELLITUS - LITERATURE REVIEW AND CASE REPORT
}

\author{
Alexis-Virgil Cochino ${ }^{1,2}$, Sigrid Covaci ${ }^{1,2}$, Oana-Maria Farkas ${ }^{1}$ \\ ${ }^{1}$ Alessandrescu-Rusescu National Institute for Mother and Child Health, Bucharest \\ ${ }^{2}$ Carol Davila University of Medicine and Pharmacy, Bucharest
}

\begin{abstract}
Glucocorticoids (GCs), one of the principal drug classes used in rheumatology, are known for their fast onset and powerful action, but also for their multiple adverse effects, the most feared ones being osteoporosis, steroidinduced diabetes (SID), cardiovascular diseases and, in children, the decrease of linear growth rate. Due to data scarcity, most SID treatment recommendations are not evidence-based. Using the minimum dose and shortest possible treatment are preventive measures generally recommended, which are frequently not followed, due to fear of GC-sparing immunosuppressive agents' side effects (DMARDs). Physiologic dose splitting, whenever possible, can be efficient in preventing at least some of the GCs' adverse effects, mainly the suppression of the hypothalamic-pituitary axis and SID. These are depicted by a case presentation, of a 12-year-old female patient with SID caused by high dose GC treatment for juvenile dermatomyositis (JDM), in whom this method was sufficient to control glycaemia.
\end{abstract}

Keywords: steroids, glucocorticoids, diabetes, dose splitting

Glucocorticoids (GCs), synthetic analogues of adrenal glands' hormones, are one of the most frequently used drugs in rheumatology. Their advantage is the fast onset, with almost immediate effect on inflammatory signs.

While using GCs (as with any other treatment), the goal is to obtain the highest benefit with the mildest (or no) adverse effects. As far as daily repartition of doses is concerned, this should fulfil two conditions: to efficiently control the disease activity and to simulate the endogenous synthesis of GCs (in order to minimize side effects). The latter, which takes place in the reticular part of the adrenal glands under the control of the hypothalamic-pituitary axis, peaks in the morning, with later decline over the day. Thus, in order to simulate this pattern, the highest GC level should be obtained in the morning. This can be done either by using the highest dose of fastmedium-action compounds (prednisolone, prednisone) in the morning, or by using an evening dose of slow acting ones (such as deflazacort). As the most frequently used GCs are prednisolone and prednisone, the former method is mostly used. Regarding the first condition, at least in rheumatoid/juvenile idiopathic arthritis, physiologic splitting of doses conveniently overlaps with symptoms flaring in the morning.

GCs' many adverse effects warrant conveniently fast weaning when the drugs are tapered, which is to be done as soon as the partial/total remission has been achieved, while maintaining it with other drugs (disease-modifying anti-rheumatic drugs, DMARDs). The most feared side effects of GCs are osteoporosis, steroid-induced diabetes mellitus and cardiovascular diseases and, in children, also impaired linear growth (1). These seem to occur more frequently in certain individuals, possibly related to some genetic background involving the GC receptors (2). Nevertheless, the cause is mostly iatrogenic, related to prolonged therapy, high dose, and its splitting over the day.

Steroid-induced diabetes mellitus represents hyperglycemia occurring in a patient previously unknown to have diabetes. Risk factors are obesity, acanthosis nigricans, family history of diabetes, preexisting low tolerance to glucose, high dose or prolonged GC therapy (3). Hyperglycemia is mostly postprandial and it is due to increased glucose production, decreased periphery uptake, decreased storage as glycogen; these are the effects of mechanisms 


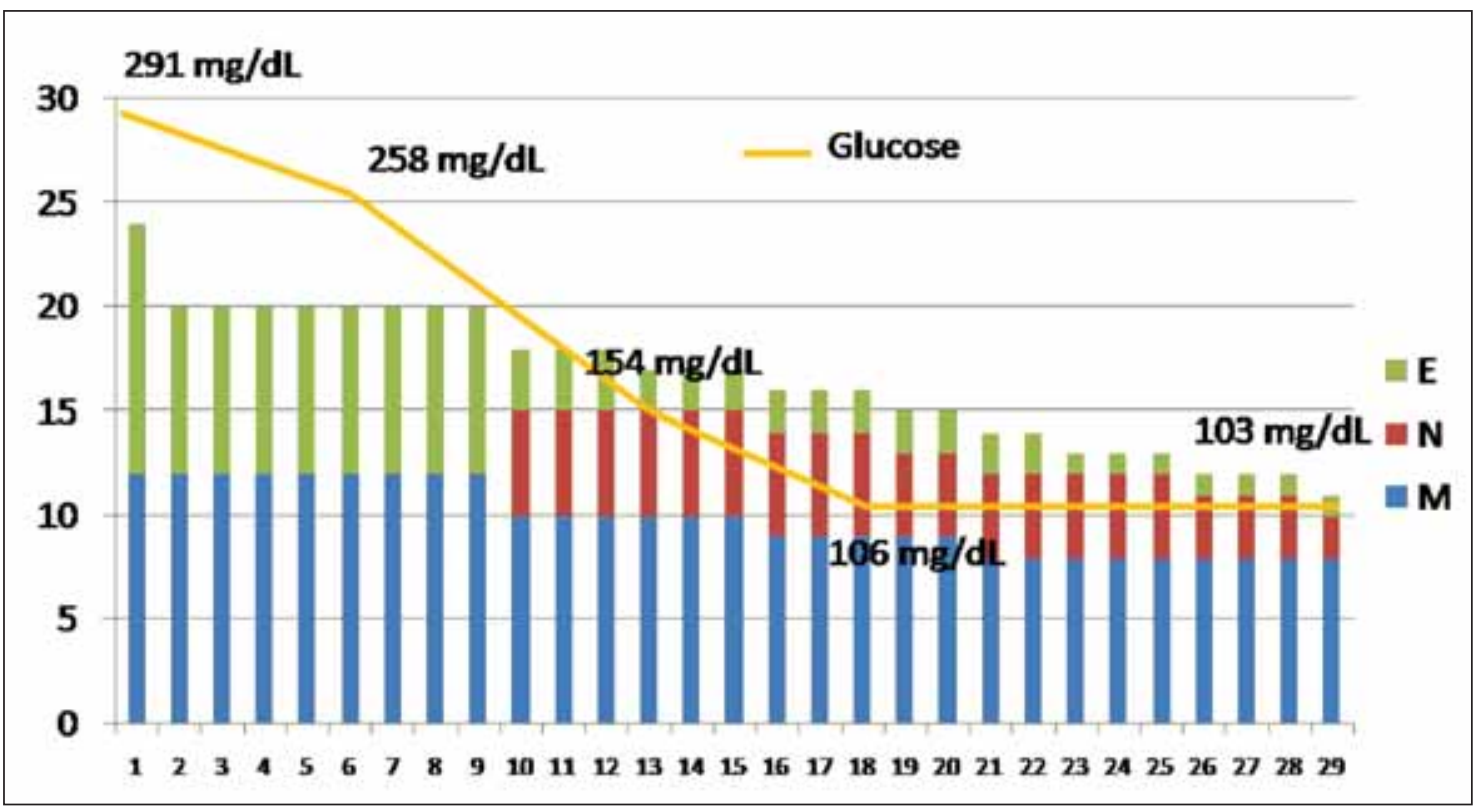

FIGURE 1. GC dose/day ( $M=$ morning, $N=$ noon, $E=$ evening) and blood glucose level

involving the pancreatic beta islet cell, hepatocyte, and muscle cell.

Available data is scarce and, as such, current treatment guidelines are mostly not evidence-based (4). They recommend diet change and insulin treatment (4-6), somehow neglecting the obvious and most probable intervention to have effect, i.e. GC weaning; this is probably due to the assumption that the shortest time and lowest dose will always be used, unless disease activity will not permit this. However, authors' experience is that fear of immunosuppressive (DMARDs), amongst both doctors and patients, is such that a "little steroid-abuse" will be preferred, against the use of a DMARD. At least in pediatrics, this is probably due to lack of experience in using them (notably methotrexate and hydroxychloroquine), more than real serious possible side effects (such as those of cyclophosphamide or rituximab, for example). Meanwhile, the very same cases prove that "steroid - phobia" is also a concern, as less evidence-based and less efficient ways of preventing GC side effects are employed (e.g. alternate day regimen).

Thus, high dose, prolonged therapy and unphysiological splitting of doses may and do cause avoidable side effects.

We therefore present the case of a 12-year-old female patient, diagnosed in January 2016 with JDM and being treated for this with prednisolone $80 \mathrm{mg} /$ day (since Jan 2016) and methotrexate (since March 2016). There is no data about glycaemia for Jan-May
2016, except for one value of $120 \mathrm{mg} / \mathrm{dL}$ in March 2016. Due to lack of disease control, and new-onset hyperglycemia, she is referred for further management, while on $80 \mathrm{mg}$ of prednisolone per day (equally split in morning and evening doses) and having glycaemia of 291-321 mg/dL. The main short term goal was to control hyperglycemia, this most probably being iatrogenic steroid-induced diabetes mellitus. In order to more easily finely tune the GC dose, prednisolone (16 mg/unit) was replaced with prednisone (5 mg/unit), at an initial equivalent dose (100 mg/day). Based on physiology notions and also on authors' own experience of GC treatment, including high dose and prolonged duration in more than 100 children with autoimmune and autoinflammatory diseases in whom no serious side effects were encountered (at least as far as glycemic control is concerned), the dose was split morning-noon-evening in 55\%-27\%-18\%. Subsequently, dose was tapered by steps of $5 \mathrm{mg}$ prednisone every 3 days, while trying to maintain the physiologic splitting pattern. It should be mentioned that at admission the patient was on very low energy intake $(\sim 1000 \mathrm{kcal} /$ day), out of which $\sim 35 \%$ were carbohydrates. Even though her diet was corrected to contain 1600-2200 $\mathrm{kcal} /$ day, glycaemia dropped to $154 \mathrm{mg} / \mathrm{dL}$ in the $4^{\text {th }}$ day ( $95 \%$ of initial prednisone dose) and to $104 \mathrm{mg} /$ $\mathrm{dL}$ in the $9^{\text {th }}$ day $(90 \%$ of initial prednisone dose) (Fig.1). Subsequent glycaemia remained normal, while patient's general state, appetite and muscular force gradually improved, possibly unmasking other 
GC side effects also present; this aspect, however, is beyond the scope of the present paper.

The goal of this communication is to very briefly review literature data on steroid-induced diabetes mellitus and, through a case presentation, to show the importance of physiological splitting of GC doses in preventing adverse effects, at least as far glycemic control is concerned.

\section{REFERENCES}

1. van der Goes M.C., Jacobs J.W., Boers M., et al. Patient and rheumatologist perspectives on glucocorticoids: an exercise to improve the implementation of the European League Against Rheumatism (EULAR) recommendations on the management of systemic glucocorticoid therapy in rheumatic diseases, Ann Rheum Dis 69:1015- 1021, 2010.

2. Firestein, Gary S., William N. Kelley. Kelley's Textbook of Rheumatology. Philadelphia, PA: Elsevier/Saunders, 2013.

3. Clore J.N., Thurby-Hay L. Glucocorticoid-induced hyperglycemia. Endocr Pract. 2009 Jul-Aug; 15(5):469-74. doi: 10.4158/EP08331. RAR.

4. Kiran Kumer, Tabita Randall, Louise Denvir, et al. Guideline for Initial management of steroid induced hyperglycaemia
- Nottingham Children's Hospital - Notthingam University Hospitals, December 2014. www.nuh.nhs.uk/handlers/downloads. ashx?id=61074. Accessed on $20^{\text {th }}$ of June, 2016

5. Liu D., Ahmet A., Ward .L, et al. A practical guide to the monitoring and management of the complications of systemic corticosteroid therapy. Allergy, Asthma, and Clinical Immunology: Official Journal of the Canadian Society of Allergy and Clinical Immunology. 2013; 9(1):30. doi:10.1186/1710-1492-9-30.

6. Hwang J.L., Weiss R.E. Steroid-induced diabetes: a clinical and molecular approach to understanding and treatment. Diabetes/ metabolism research and reviews. 2014; 30(2):96-102. doi:10.1002/dmrr.2486. 